\title{
Assessing patient usability of video visits
}

\author{
Milan Patel ${ }^{1,2}$, Rebecca Miller ${ }^{3}$, Haley Haddad $^{3}$, Larry An $^{1,3,4}$, Jessie Devito $^{3}$, Alison Neff ${ }^{3}$, Abishek Rajkumar ${ }^{1,2}$, \\ Chad Ellimoottil ${ }^{1,3,5}$
}

${ }^{1}$ Institute for Healthcare Policy and Innovation, University of Michigan, Ann Arbor, MI, USA; ${ }^{2}$ University of Michigan Medical School, Ann Arbor, MI, USA; ${ }^{3}$ Michigan Medicine Virtual Care Team, University of Michigan, Ann Arbor, MI, USA; ${ }^{4}$ Department of Internal Medicine, ${ }^{5}$ Department of Urology, University of Michigan Medical School, Ann Arbor, MI, USA

Contributions: (I) Conception and design: All authors; (II) Administrative support: C Ellimoottil; (III) Provision of study materials or patients: R Miller; (IV) Collection and assembly of data: M Patel, R Miller, C Ellimoottil; (V) Data analysis and interpretation: M Patel, C Ellimoottil; (VI) Manuscript writing: All authors; (VII) Final approval of manuscript: All authors.

Correspondence to: Milan Patel. University of Michigan, 2800 Plymouth Rd., Bldg 16, 1st Floor, Room 152S, Ann Arbor, MI 48109, USA.

Email: milankp@med.umich.edu.

Background: Telehealth is being adopted by health systems across the country and many barriers to the expansion of video visit programs have been identified. Our study focused on the usability of video visit technology by examining technical challenges faced by patients over the course of a video visit.

Methods: We conducted a survey of patients who received care from the Michigan Medicine video visit program from January 31, 2019 to July 31, 2019. The video visit program includes more than 1,300 visits a year across more than 30 specialties. Following the completion of their video visit, all patients were invited to participate in our online survey through the patient portal. The survey included questions on patient satisfaction, motivation and technical challenges.

Results: We received responses from 180 patients (response rate of 26\%). Overall patient satisfaction was high; $90 \%$ of respondents agreed that their video visit experience was similar to that of in-person visits and 93.3\% of respondents would recommend video visits. Despite this high satisfaction rate, 36 out of $180(20.0 \%)$ respondents cited technical issues during their video visit: video issues $(n=11)$, audio issues $(n=5)$, video and audio issues $(n=2)$, slow/dropped connection $(n=7)$, initial set-up issues $(n=4)$, long wait time $(n=3)$, and other $(n=4)$.

Conclusions: While most patients report a high degree of satisfaction with their video visit, a meaningful subset of patients continue to experience technical challenges.

Keywords: Telehealth; mHealth; eHealth; telemedicine

Received: 08 January 2020; Accepted: 21 August 2020; Published: 20 April 2021.

doi: $10.21037 /$ mhealth-20-30

View this article at: http://dx.doi.org/10.21037/mhealth-20-30

\section{Introduction}

The use of telehealth is expanding into many areas of medicine, ranging from sleep medicine to addiction treatment to pediatric cardiology (1-3). With advances in technology, more institutions are recognizing the advantages of providing healthcare remotely. Simultaneously, broader population (consumer) adoption and the use of the internet and mobile communication technology has led to telehealth becoming a viable healthcare option for an increasing number of patients.
For example, $73 \%$ of U.S. adults currently have access to high-speed broadband service at home, compared to only $24 \%$ of U.S. adults 15 years ago (4). Nevertheless, a wide range of obstacles inhibit the implementation and expansion of telehealth, including systemic and logistical challenges such as liability, licensure, reimbursement, and workflow management (5). Recently, more attention has been directed at investigating patient-specific factors, as demonstrated in a systematic review which found age, level of education, 
and lack of computer literacy to be frequent barriers to the adoption of telehealth (6).

Nevertheless, these studies are limited to describing issues with the patient population in general rather than with the experiences of patients who are receiving telehealth services. For instance, despite technological advances in the use of video visits (a form of telehealth), poor visual and/or audio quality may hinder the video visit experience for many patients. This aspect of the patient experience may be unknown to telehealth programs, as it is widely assumed that the technology underlying these telehealth programs are of high quality and unlikely to cause disruptions. However, if institutions are made aware of this hindrance, they can direct their attention to ensuring that their services are a clear and effective delivery of medicine from a distance. Given the broad uptake of video visits, it is important to understand concerns raised by patients in order to improve patient satisfaction and guide the creation of new programs.

Select studies in this area have begun identifying some of the technical issues with the patient experience of telehealth services, but the data is limited (7-9). Herein, our study takes a novel approach by centering our investigation on the technical challenges surrounding video visits. This study was intended to be hypothesis-generating in order to drive quality improvement of a video visit program. We present the following article in accordance with the SURGE reporting checklist (available at http://dx.doi.org/10.21037/ mhealth-20-30).

\section{Methods}

We collected our data from the Michigan Medicine video visit program, which was established in 2016. The video visit program includes more than 1,300 visits a year across more than 30 specialties. The Michigan Medicine video visit program defines a video visit as a synchronous (live, 2-way audiovisual) face-to-face, scheduled visit between a Michigan Medicine patient at home and a Michigan Medicine provider. Michigan Medicine uses Epic software coupled with Vidyo for its video visit program. To participate in a video visit, a patient is asked to download and access the patient portal mobile app via a smartphone or tablet and confirm that they will have a strong wireless or cellular data connection for the visit. While a laptop or desktop connection is possible, it is not recommended to the patient because of the increased likelihood that there will be a technical challenge related to the connection. Additionally, patients are asked to complete some preparation and setup prior to their video visit including testing the connection via the patient portal mobile app at least 1 day before the appointment and following instructions to complete an e-Check-In 15 to 30 minutes before the appointment.

All patients in this study received an electronic survey following the completion of their video visit. The survey, which included questions on technical challenges, patient satisfaction, and motivation, was administered online through Qualtrics and was sent via the patient portal to all patients who had a completed video visit from January 31, 2019 to July 31, 2019. No additional attempts were made to request survey completion from patients and no financial or other incentives were offered for completion of the survey.

The primary objective of the survey was to understand the technical challenges faced by patients over the course of a video visit. The survey featured a multiple-choice question with select pre-determined categories for technical issues in addition to a free-response option for patients who selected "Other" to the initial question. The predetermined categories were "No technical issues", "Slow or dropped connection", "Audio issues", and "Video issues". Additional categories were created to incorporate free-text responses to "Other". In addition to patient challenges, our secondary outcomes included patient satisfaction and patient motive for choosing video visits. Patient satisfaction was assessed in two ways. First, we asked patients if their video visit was similar to an in-person visit. Second, we asked if patients were likely to recommend video visits. Patient motive for choosing video visits was assessed via response to a multiple-choice question with select pre-determined categories. These categories included "No travel", "Saves Times", "Easier to fit in my schedule", "Shorter wait for an appointment", "Followed provider's recommendation", "Someone else would need to take me to an in-person visit", and "Other". Descriptive statistics were used to analyze the categorical responses to our survey questions.

The study was conducted in accordance with the Declaration of Helsinki (as revised in 2013). This study was deemed exempt from review by the University of Michigan Institutional Review Boards (IRB) as it was focused on quality improvement. Study participants were not required to provide informed consent as the study was voluntary, anonymous, and posed minimal risk.

\section{Results}

During our study period, 730 video visits were scheduled 
Table 1 Key results of video visit patient survey

\begin{tabular}{lc}
\hline Question & No. of patients $(\%)$ \\
\hline $\begin{array}{l}\text { 1. Did you experience any technical issues with your video visit } \\
\text { today? }\end{array}$ & $11(6.1)$ \\
Video issues & $5(2.8)$ \\
Audio issues & $2(1.1)$ \\
Video and audio issues & $7(3.9)$ \\
Slow/dropped connection & $4(2.2)$ \\
Initial set-up issues & $3(1.7)$ \\
Long wait time & $4(2.2)$ \\
Other & $36(20.0)$ \\
Total &
\end{tabular}

2. Was your video visit experience similar to care you expect to receive at Michigan Medicine?

$\begin{array}{lc}\text { Yes } & 162(90.0) \\ \text { No } & 6(3.3) \\ \text { Unsure } & 12(6.7)\end{array}$

3. Would you recommend video visits following this experience?

$\begin{array}{lc}\text { Yes } & 168(93.3) \\ \text { No } & 6(3.3) \\ \text { Unsure } & 6(3.3)\end{array}$

4. Why did you choose to have a video visit versus an in-person visit?

$\begin{array}{lc}\text { No travel } & 113(62.8) \\ \text { Saves time } & 97(53.9) \\ \text { Easer to fit in my schedule } & 92(51.1) \\ \begin{array}{l}\text { Followed provider's } \\ \text { recommendations }\end{array} & 89(49.4) \\ \text { Shorter wait for an appointment } & 38(21.1) \\ \begin{array}{l}\text { I prefer virtual care/l did not want to } \\ \text { come in-person }\end{array} & 17(9.4) \\ \begin{array}{l}\text { Someone else would need to take } \\ \text { me to an in-person visit }\end{array} & 17(9.4) \\ \text { Other } & 23(12.8)\end{array}$

and 680 video visits were successfully completed through the Michigan Medicine video visit program. We collected survey responses from 180 patients with a response rate of $26 \%$ (180/680). Providers were from both primary care and several specialties including urology, gastroenterology,
Table 2 Patient quotes describing technical challenges

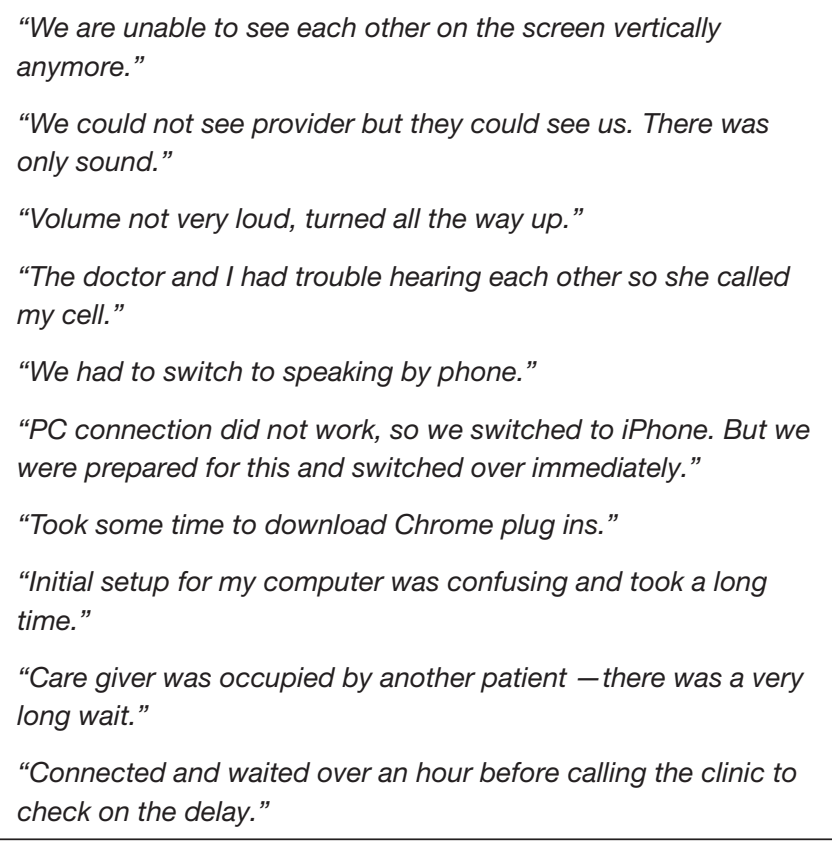

general surgery, and psychology. Sixty-one percent of the patients in our sample population were female and $39 \%$ were male. Patients in our sample population ranged in age from 2 months old to greater than 90 years old, with the average age being 40.3 years old. Because our survey was anonymous, we were not able to calculate descriptive statistics for our respondent population. The full distribution of responses to these questions are depicted in Table 1.

In regards to patient satisfaction, 162 out of 180 (90.0\%) respondents to the survey answered "Yes" to the question: "Was your video visit experience similar to care you expect to receive at Michigan Medicine?" One hundred and sixtyeight out of 180 (93.3\%) respondents answered "Yes" to the question: "Would you recommend video visits following this experience?"

Overall, 36 out of $180(20.0 \%)$ responses reported a technical issue with their video visit. We categorized these issues into the following seven groups: video issues $(n=11)$, audio issues $(n=5)$, video and audio issues $(n=2)$, slow/ dropped connection $(n=7)$, initial set-up issues $(n=4)$, long wait time $(n=3)$, and other $(n=4)$. Select patient statements describing technical challenges can be seen in Table 2 .

When asked "Why did you choose to have a video visit versus as in-person visit?", patients were given the option 
to choose up to eight different motives. The following four motives received the most votes: "No Travel" $(\mathrm{n}=113)$, "Saves Time" (n=97), "Easier to fit in my schedule" ( $\mathrm{n}=92)$, and "Followed provider's recommendations" $(\mathrm{n}=89)$. The remaining four motives were "Shorter wait for an appointment" ( $\mathrm{n}=38)$, "I prefer virtual care/I did not want to come in-person" ( $\mathrm{n}=17)$, "Someone else would need to take me to an in-person visit" ( $\mathrm{n}=17)$, and "Other" $(\mathrm{n}=23)$.

\section{Discussion}

In this study, we found that while a vast majority of patients experience no technical issues with their video visit, a meaningful subset of patients does report some form of technical difficulty, the most common of which is related to visual quality. As a whole, patients in our sample size were satisfied with their video visit, agreeing that it was on-par with the quality of an in-person visit and willing to recommend video visits in the future. Furthermore, the most common reasons that patients chose video visits over in-person care were that video visits allowed them to avoid travel and save time. Collectively, these findings suggest that while patients are largely satisfied with the quality of care delivered over video visits, basic technical issues continue to be an area of concern.

Our findings that one in five patients still have technical issues with their video visit experience is consistent with other studies. For instance, Powell et al. also utilized a postvisit patient survey and discovered that although patients did have high rates of satisfaction, technical issues were by far the predominant cause of dissatisfaction (10). Both Sturesson et al. and Taylor et al. identified challenges in the areas of audio quality and video quality in their respective studies, though they gathered their data from provider feedback, rather than patient feedback $(8,9)$. With these collective findings in mind, it is imperative for institutions to create measures to get around these technical issues. Interestingly, the physician is often prompted to provide immediate and unrehearsed technical support when an issue arises over the course of a video visit, but this initial help is understandably limited (8). To fill this void, we have established a dedicated patient-facing technical support team at Michigan Medicine. However, the results of this study also demonstrate that we can improve the patient experience by implementing changes prior to the start of the video visit. Many of the technical challenges experienced by patients that were related to initial-set up or fell in the "Other" category may have been avoided if the patient utilized the recommended mobile app interface as opposed to an internet browser. Thus, this magnifies the importance of increasing patient education surrounding both access and video visit resources at the time of scheduling the video visit.

Our findings of high patient satisfaction are consistent with the reports of prior studies in the existing telehealth literature. For example, in another study of multispecialty telehealth delivery, an average of $4.8 / 5$ on the Likert scale was reported for patient satisfaction (11). Thus, it is not surprising that a high number of patients in our study said they would recommend video visits in the future. However, the success of video visits is more accurately gauged when assessing their efficacy against in-person visits. Importantly, only $1 / 3$ of studies in a systematic review of 93 telehealth studies were found to report such a measure of comparison (12). Our study addressed this need and found that an overwhelming majority of patients did indeed feel that their video visit met the standard of care delivered via in-person care.

It has also been well-established in the literature that convenience is likely the most appealing quality of video visits. The time-saving nature of video visits, in particular, is frequently cited $(10,13)$. It has been found to be attractive not only to patients and physicians, but also to other healthcare providers such as nurses and technicians (14). When focusing on efficiency, the exact time saved is often substantial—one study reported $40.8 \%$ of patients saving more than three hours of time by utilizing video visits (10). Our findings reinforce the value of convenience, as the top three reasons for choosing video visits in our study were all related to convenience, with the second most common being directly related to time efficiency. Our work also expands upon the literature in identifying a new and fairly unique patient motive: almost half of our patients reported provider recommendation as playing a role in their decision to engage in video visits.

There are several limitations to this study. First, our study was performed at a single institution, which may limit the generalizability of results across different populations. However, our patient population was diverse with respect to age and specialty of care received. Another limitation of our study is that we inquired about technical challenges by asking a multiple-choice question in which responses were limited to pre-set options. However, we did include an option categorized as "Other"; if this option was selected, respondents were asked an additional follow-up question for further explanation. A third limitation is that we only achieved a response rate of $26 \%$. Nevertheless, we feel that despite our low response rate, we were able to identify a 
pattern of technical challenges that is likely generalizable to our entire patient population. A fourth limitation is that our study does not include patients who were scheduled for video visits but were unable to receive care because they experienced profound technical difficulties. We are fairly certain this pool of patients exists based on anecdotal evidence from our virtual care staff. However, the data from these patients would only increase the prevalence of technical issues among video visits and further support our conclusion.

These limitations notwithstanding, our study has important implications for health systems, physicians, and patients. For health systems, our findings promote the integration of technical standards for audio quality, visual quality, and connectivity (though not limited to these factors). Our study further suggests that current health systems should proactively seek feedback from patients to ensure that technical standards are being met, and that patient feedback regarding technical standards should be obtained early in the launch of new programs. A combination of a patient-facing technical support team and increased patient education about video visits may be the ideal solution to accomplish both these tasks. For physicians, our study indicates that when the proper technical standards are met, physicians should be confident in their ability to deliver effective medical care, as patients generally find the quality of video visits to be equivalent to that of in-person visits. Thus, for patients, our findings encourage changes that will both enhance the experience for current users and invite new patients to engage in video visits.

\section{Conclusions}

In summary, this study demonstrates that even with established video visit programs, technical challenges such as video, audio, and connectivity issues can still persist. Therefore, it is recommended that large healthcare institutions that are currently implementing video visits have support available for patients to overcome these challenges. Moreover, future work in this area should extend beyond the limits of technical issues and investigate other patient concerns with video visits such as security and cost implications relating to insurance coverage. With continued focus on improving the patient's experience with video visits and other forms of telehealth, this exciting and rapidly growing modality of healthcare delivery will continue to revolutionize the field of medicine.

\section{Acknowledgments}

Funding: This work was supported by the Telehealth Research Incubator project grant (MPrOVE Research Challenge Grant).

\section{Footnote}

Reporting Checklist: The authors have completed the SURGE reporting checklist. Available at http://dx.doi.org/10.21037/ mhealth-20-30

Data Sharing Statement: Available at http://dx.doi. org/10.21037/mhealth-20-30

Conflicts of Interest: All authors have completed the ICMJE uniform disclosure form (available at http://dx.doi. org/10.21037/mhealth-20-30). CE serves as an unpaid editorial board member of $m$ Health. The other authors have no conflicts of interest to declare.

Ethical Statement: The authors are accountable for all aspects of the work in ensuring that questions related to the accuracy or integrity of any part of the work are appropriately investigated and resolved. The study was conducted in accordance with the Declaration of Helsinki (as revised in 2013). This study was deemed exempt from review by the University of Michigan Institutional Review Boards (IRB) as it was focused on quality improvement. Study participants were not required to provide informed consent as the study was voluntary, anonymous, and posed minimal risk.

Open Access Statement: This is an Open Access article distributed in accordance with the Creative Commons Attribution-NonCommercial-NoDerivs 4.0 International License (CC BY-NC-ND 4.0), which permits the noncommercial replication and distribution of the article with the strict proviso that no changes or edits are made and the original work is properly cited (including links to both the formal publication through the relevant DOI and the license). See: https://creativecommons.org/licenses/by-nc-nd/4.0/.

\section{References}

1. Zia S, Fields BG. Sleep Telemedicine: An Emerging Field's Latest Frontier. Chest 2016;149:1556-65.

2. Molfenter T, Boyle M, Holloway D, et al. Trends in 
telemedicine use in addiction treatment. Addict Sci Clin Pract 2015;10:14.

3. Satou GM, Rheuban K, Alverson D, et al. Telemedicine in Pediatric Cardiology: A Scientific Statement From the American Heart Association. Circulation 2017;135:e648-78.

4. Demographics of Internet and Home Broadband Usage in the United States. Pew Research Center: Internet, Science \& Tech. Available online: https://www.pewinternet.org/ fact-sheet/internet-broadband/ (2019, accessed 4 August 2019).

5. Gordon J, Tsay D, Coyne S, et al. Four Challenges of Launching a Telehealth Program. NEJM Catalyst. Available online: https://catalyst.nejm.org/challengeslaunching-telehealth-program/ (2017, accessed 7 July 2019).

6. Scott Kruse C, Karem P, Shifflett K, et al. Evaluating barriers to adopting telemedicine worldwide: A systematic review. J Telemed Telecare 2018;24:4-12.

7. Powell RE, Henstenburg JM, Cooper G, et al. Patient Perceptions of Telehealth Primary Care Video Visits. Ann Fam Med 2017;15:225-9.

8. Sturesson L, Groth K. Effects of the Digital Transformation: Qualitative Study on the Disturbances and Limitations of Using Video Visits in Outpatient Care.

doi: $10.21037 /$ mhealth-20-30

Cite this article as: Patel M, Miller R, Haddad H, An L, Devito J, Neff A, Rajkumar A, Ellimoottil C. Assessing patient usability of video visits. mHealth 2021;7:22.
J Med Internet Res 2018;20:e221.

9. Taylor A, Morris G, Pech J, et al. Home Telehealth Video Conferencing: Perceptions and Performance. JMIR Mhealth Uhealth 2015;3:e90.

10. Powell RE, Stone D, Hollander JE. Patient and Health System Experience With Implementation of an EnterpriseWide Telehealth Scheduled Video Visit Program: MixedMethods Study. JMIR Med Inform 2018;6:e10.

11. Waibel KH, Cain SM, Hall TE, et al. Multispecialty Synchronous Telehealth Utilization and Patient Satisfaction Within Regional Health Command Europe: A Readiness and Recapture System for Health. Mil Med 2017;182:e1693-7.

12. Williams TL, May CR, Esmail A. Limitations of patient satisfaction studies in telehealthcare: a systematic review of the literature. Telemed J E Health 2001;7:293-316.

13. Tasneem S, Kim A, Bagheri A, et al. Telemedicine Video Visits for patients receiving palliative care: A qualitative study. Am J Hosp Palliat Care 2019;36:789-94.

14. Ditchburn JL, Marshall A. Renal telemedicine through video-as-a-service delivered to patients on home dialysis: A qualitative study on the renal care team members' experience. J Ren Care 2017;43:175-82. 